\title{
Case Study on Scientific Researcher's Motivation at TF Institute
}

\author{
Hongzhen Deng \\ School of Business, Jianghan UniversityWuhan, P.R. China \\ denghong13@126.com
}

Keywords: Scientific researcher; Staff's motivation; TF institute

Abstract. This paper takes the scientific research staff of TF Institute as the object, and aims at putting forward detailed improvement by analyzing the current situation and existing problems of employee motivation of researchers in TF institute.

\section{Introduction}

In today's increasingly competitive era of knowledge economy, the contest of science and technology is, in the long run, a contest of talents. Whoever can fully arouse the motivation of the staff, and stimulate their efforts to work independently will be able to take the advantages in the contest.

Although there are many theories about employee motivation over the world, such as Need-hierarchy theory, Two factor theory, Equity theory, Reinforcement theory, etc. But the research object is always confined to universal category and research on specific person are not in-depth and system. Therefore, how to develop the motivation systems in accordance with the organization's feature under the guidance of theoretical research by combining the theory with the characteristics of knowledge workers, and with the organization's management practice is worthy of in-depth study.

\section{Case Background of TF Institute}

The TF Institute was founded in 1950, it is a research institute engaged in designing of the special air vehicle. In 1990s, the business of TF Institute turns around by the development of civilian industry and civil aviation which have made a great progress. In the twenty-first Century, new opportunities for development appear with the adjustment of the national industry, part of its original business has become the focus of the group's development strategy after a comprehensive integration of the whole business within the group. Its general aviation business, special aircraft research and development business have been switched to the group's key support, and new research and development centers have been set up in coastal cities.

So far, TF Institute has 1400 employees, including 1100 scientific researchers, 150 managers, and 150 auxiliary production workers. 30\% of the scientific researchers hold the degree of master and above.

From the geographical point of view, $50 \%$ of the researchers majorly work in the head institute, and the rest work in the Zhuhai branch Institute. The overall quality of staff is high and the age is about 35 years' old which is in the golden age for technological productions. Notably, only the researchers working in the head institute are involved in this research considering the significant differences between management mechanism of the Zhuhai branch institute and the capital institute.

\section{Current Status of Staff's Motivation of TF Institute}

A great deal of active explorations has been made by the TF Institute to stimulate staff's work enthusiasm, thusly improve the work efficiency and ensure the completion of the model development task on schedule. These explorations mainly including the improvement of the salary system, paying attention to the award of the achievements, implementation of task award, topic model development award and other types of incentives. Besides, careless for the life of staff, carrying out rich and colorful leisure activities, careless for staff health, regularly organization of staff physical examination have been performed. The implementation of a series of systems as mentioned above, ensure the employees' 
income, meet the needs of employees' work and life to a certain extent which have a certain attraction for talent and result in a relatively stable staff structure.

But the enthusiasm of researchers is still not high enough. Some employees shop online, play mobile phone games in office works. Employees work exactly according to the work timetable and are unwilling to work overtime even the tasks are unfinished. The task is always later than scheduled deadline or completed with low quality. Employee has turned a blind eye on these questions.

Questions from the Top Manager. The TF Institute's top management team is an excellent team. They have exceptional strategic planning capabilities and can develop appropriate development strategies based on changes in the situation and the Institute's reality. This is a tough, innovative team. Guided by the idea of the out of thin air, they realize the civilian products and the model development growing out of nothing.

However, the survey shows that employees have the lowest satisfaction with senior managers, mainly due to the lack of high-level managers' communication with staff on strategic planning, resulting in employees' failure to understand.

The senior managers treated the motivation of scientific research staff are too simply for lacking of the understanding of scientific research personnel motivation. They only emphasize on the salary motivation by ignoring the promotion of the system of staff training and management. They prefer to take short, frequency, fast actions and are not willing to consider the problem systematically.

Difficulties in the Designing of the Salary System. Perfect compensation system has been the focus of attention of mangers of TF. The employee income of TF Institute is in an absolutely leading position locally after three salary reforms in 8 years (income growth rate is higher than the $30 \%$ for each time). Senior leaders of TF institute hopes to stimulate the work enthusiasm of the staff to improve their work efficiency and promote the scientific research tasks by simply increasing the employee's income. Things always turn out contrary by the fact that each reform caused major mood fluctuations of the staff. The main reason lies in:

The salary system design is not fully integrated with job management. The design of scientific researchers' growth channel is insufficient. Although it emphasizes the separation of "leader" and "institute", the management path is still higher than the technical path in post setting. And the lack of the design to post competency and the mechanism to evaluate cognitive ability result in a fixed staff positions for a long time once identified in the pay reform, which cannot be adjusted in a timely manner.

The lack of systematic view of the remuneration system leads to the failure of the smooth operation of system and finally lack of confidence in management team. The salary system is the foothold of all kinds of management and the final embodiment of management will. The smooth operation of the remuneration system needs to be organically integrated with and supported by the post management system, competency evaluation system, occupation development planning system, performance appraisal system, model management system, project management system. Only in this way, can the salary management idea realize.

Only compensation system was performed in the salary reform in 2010 of TF Institute, and lack of other following management systems, resulting in that the salary cannot be dynamic linked with the position, performance. No one can be punished at the end of the year, nor can anyone be rewarded.

Problems Existed in Performance Evaluation System. Knowledge workers have a high need for achievement, and the research work is not easy to be measured. The features of long period and high risk of scientific research make it difficult to be scientifically evaluated. And then scientific evaluation of the employee performance is the difficulty in the motivation of knowledge staff.TF Institute is more backward in the examination and excitation of scientific researchers. The first is that the decomposed work mechanism of the strategic objectives is not formed, resulting in only a few people is familiar with the strategic objectives of the organization. And also the strategy is not applicable because that the strategy only stays in the strategic level without corresponding action plan and evaluation index of the strategy.

Secondly, there is no effective performance appraisal system. Though TF Institute has developed a reward system for models, projects and revenues generating tasks, mismatched and uncoordinated 
systems cannot operate properly. And also there is no assessment system for employees. So the completion of the tasks cannot be docked with individuals.

\section{Incentive Countermeasure of Scientific Researchers}

We understood the current motivation situation of researchers in TF Institute through above-mentioned analysis, and found that there is greater room for the improvement of the incentive of TF Institute researchers. First of all, we need to establish a correct view of motivation and treat the scientific researchers' motivation with a systematic and scientific view point. The investigation shows that the main factors that influence the job engagement of the scientific researchers in TF Research Institute are top managers, career development, performance management, intrinsic motivation and salary. Considering the satisfaction of various factors, we determined that the first problem need to be solved was executive management, compensation and performance management. In this paper, recommendations on the design of TF Institute incentive system and detailed specific solutions were proposed based on top manager, compensation, and performance appraisal.

Improve the TF Institute Incentive System. Some methods can be adopted to Improve the TF Institute Incentive System as follow:

Scientific Designing of Incentive System. Firstly, the organizational incentive system need to be designed on the basis of fully understand of motivation theory under the guidance of scientific theory. A few of people desired for highly successful in the system host a strong sense of self-motivation. Such people need to be highly concerned and polices and stage to fully release their potential. Most people need incentive to improve performance. In the system design, we should make full use of the demand theory and reinforcement theory, so as to ensure that the designed incentive system conforms to the objective law and can operate effectively. Secondly, there must be a scientific workflow. It is a common mistake in the design of incentive system to take the will of leadership as the flag, and to determine the direction of improvement with subjective assumptions. Scientific processes are necessary to achieve scientific results.

Systematical Incentive System Design. As a complex project, we cannot expect employee motivation to be solved only relying on one or several systems. To design incentive system, we have to fully consider the various needs of employees, draw up incentive system network, and build up this network according to the network planning. At the same time, the incentive should be directed at all staff while making the key point stand out, and the enthusiasm of all levels and aspects should be mobilized.

Synergetic Effect of Material Motivation and Spiritual Motivation. At present, the first thought is money when some managers talk about employee motivation, and they think that money can solve any problem. Otherwise, the lust of human is endless. It is easy to form an incentive culture that obey anyone who feeds him. While spiritual stimulation is a heart move, and has the virtues of persistence, tenacity and resonance compared with that of material incentive. Application of the spirit encouragement to work will exert a subtle influence on the results.

Combined needs. Organizations should be committed to build a positive incentive culture, to guide the staff needs to a positive cultural atmosphere through the construction of corporate culture and other ways, meanwhile meeting the basic staff needs.

Strengthen Art Practice, Improve Staff's Satisfaction to Top Leader. The survey showed that leadership scores higher in capability while lower in communication with employees, respect for employees, caring about employee benefits. So that employees cannot fully understand the intention of the leadership and sense respect from leaders so the leader cannot get the trust of employees. Several measures as followed should be taken to improve this problem.

More communications to meet mutual desire. A good leader should be a good preacher who is able to portray a better vision for the organization, to convince employees with the vision, to encourage employees to strive for the realization of vision. Therefore, senior managers need to organize strategic presentations through multiple channels, so that employees can see the future and understand the thinking of senior managers. When necessary, the leader should organize employee representatives to 
participate in the discussion before and after the release of the strategy to further unify the understanding.

Communication is the key to the success of an organization. If employees fail to understand the vision of the organization, then it is impossible for them to understand the organization's strategy for achieving this vision. Without the understanding of vision and strategy, employees cannot effectively support strategy implementation in their daily work.

The leadership team should use all possible channels for strategic communication and continue to strengthen the strategy with all possible opportunities. Commonly used communication channels include monthly summary meetings, seminars, conferences, online announcements, reports, individual exchanges, etc.

Discard authority and value employee's intelligence. Scientific research is a work which needs not only experience but also innovation. After more than 50 years of development, TF Institute has trained a number of experts with considerable work experience. But the experts cannot be blindly regarded as authority considering rapid progress of science and technology. Particularly, the science and technology needs to be treated with a changing perspective, an innovative approach, and an explorative mind. Young employees have an active mind, a strong ability to accept new things and have strong creative ability. 80\% TF Institute researchers are in the golden work age of 25-45 years. Senior leaders should constantly optimize the working mechanism to make new employees fully express their ideas and explore actively.

Caring for employees. Top managers should be concerned about employees, understand the lives and aspirations of their employees, treat their employees honestly to enhance their trust and satisfaction with top managers and organizations. TF Institute is an old institution with unique advantages in organizational structure. The organization should fully utilize the ideological fortress effect embodied departments including trade unions, the Communist Youth League and all levels of Party branch to deliver the care of the organization to all employees. And listen to the voices of employees and solve them timely.

Establish Strategy-based Fair Salary System. The survey found that employees are generally dissatisfied with the salary determination mechanism and the salary structure. The analysis shows that the salary system of TF Research Institute is flawed in fairness. Compensation itself has dual functions of health care and motivation. If the salary only stay at the wage level, is not closely linked with the post, performance, evaluation and other mechanisms, the salary tends to health care function. Otherwise, the salary system also has a strong incentive function. The design of the compensation system should determine the competitive salary level, establish scientific and reasonable post management mechanism, comprehensively consider the balance between scientific research models, subjects based on strategy. Standardization of work processes, insurance of the fairness of the program to determine the compensation, setting up reasonable salary structure also should be included in the designing of salary system. Employees who have made outstanding achievements should be specially rewarded by unique way such as awards of model completion, project and so on.

Improve the Performance Appraisal System and Strengthen Performance Appraisal. According to the expectation theory and reinforcement theory, we find that the success performance appraisal should have three elements, target system consistent with enterprise strategy, scientific and rational performance appraisal system, and rational application of the appraisal results. Therefore, based on the framework of the formulation of strategic objectives, the top manager should transfer the mission statement from high-level organization to responsible work of staff. Top manager should establish a management idea with the center of strategy, perform necessary management reform to ensure safety landing of the strategy. Top manager also should find out both implementable key objectives which has big influence on the strategy and urgent project so be performed and using them as the key parameter of KPI of department. Then business departments or work teams should further decompose the action plan to form appropriate work plan for individuals of the team. When necessary, feasibility of the strategic and strategic plan need to be reviewed again to ensure that the strategy can continue to meet the requirements of formal development, and that process supervision is effective. Finally, improved index 
collection and feedback system need to be established and improved according to problems during the assessment process, so as to improve organizational performance.

Of course, in order to ensure that these incentives can be long-term play, so as to fully mobilize the enthusiasm and initiative of employees, senior leaders must pay much high attention to all aspects mentioned above. Senior leaders should not only fully aware of the importance of incentives, but also actively participate in the implementation of the incentive measures and construction of organization incentive system, personally push the implementation of incentive measures by solving the difficulties and problems during the promotion of the system. In addition, the senior leaders should implement countermeasures after scientific and objective analysis of the characteristics of the organization to avoid the phenomenon of acclimatized.

\section{Acknowledgment}

Thanks Prof. Bo Deng (Wuhan Textile University) for his valuable discussions and suggestions.

\section{References}

[1] H. Zeng, Evaluation and Development of Employee Engagement, first ed., Nankai University Press, 2008.

[2] B. Yang, Research on the promotion of employee engagement in Chinese enterprises: based on the organizational perspective, first ed., Capital University of Economics and Business Press, 2012.

[3] Y. Cai, Practical Handbook of Integrated Balanced Scorecard, first ed., Beijing Aviation Industry Press, 2009.

[4] W. J. Cui, L. S. Li, An empirical study of motivation preferences of scientific and technical personnel-a statistical analysis based on 2600 questionnaires from Nantong, Jiangsu Province, J. Sci. Res. 27 (2009) 588-591.

[5] W. D. Li, H. Liu, H. Y. Tao, A review of work motivation of R \& D personnel of enterprise, J. For. Eco. \& Man. 30 (2008) 35-42.

[6] L. T. Li, Y. Fan, J. L. Xue, Thinking of staff incentive mechanism based on the heterogamy hypothesis of employee, J. Soc. The. Gui. 12 (2008) 106-107. 